\title{
PH E TEMPERATURA ÓTIMOS PARA ATIVIDADE ENDOGLUCANASE DE FUNGOS EM CULTIVO SÓLIDO
}

\author{
P. A. CASCIATORI ${ }^{1}$, F. P. CASCIATORI ${ }^{2}$, J. C. THOMÉO ${ }^{3}$ e R. da SILVA ${ }^{4}$ \\ ${ }^{1,4}$ Universidade Estadual Paulista, Instituto de Biociências, Letras e Ciências Exatas, Departamento \\ de Química e Ciências Ambientais \\ ${ }^{2,3}$ Universidade Estadual Paulista, Instituto de Biociências, Letras e Ciências Exatas, Departamento \\ de Engenharia e Tecnologia de Alimentos \\ E-mail para contato: pricasciatori@yahoo.com.br
}

\begin{abstract}
RESUMO - As celulases são essenciais na produção de bioetanol a partir de biomassa agroindustrial, podendo ser produzidas em cultivo sólido, mas tendo atividade dependente de $\mathrm{pH}$ e temperatura. Neste trabalho, avaliou-se a temperatura e $\mathrm{pH}$ ótimos para atividade endoglucanase dos extratos celulolíticos produzidos pelo fungo termofílico Myceliophthora thermophila I-1D3b e pelo mesofílico Trichoderma reesei QM9414 durante cultivo sólido em bagaço de cana e farelo de trigo. A ótima atividade endoglucanase foi obtida em $\mathrm{pH} 4,5$ e temperatura igual a $45^{\circ} \mathrm{C}(80 \mathrm{U} / \mathrm{g})$ para Trichoderma reesei, sendo a enzima inativada a partir de $70{ }^{\circ} \mathrm{C}$. Para Myceliophthora thermophila, a ótima atividade endoglucanase foi obtida em $\mathrm{pH} 5,5$ e temperatura igual a $65^{\circ} \mathrm{C}(350 \mathrm{U} / \mathrm{g})$, e somente a partir de $80{ }^{\circ} \mathrm{C}$ a atividade endoglucanase declinou, sugerindo que as celulases de fungos termofílicos são promissoras para aplicação na cadeia de produção do etanol de segunda geração.
\end{abstract}

\section{INTRODUÇÃO}

A produção de enzimas celulolíticas tem despertado grande interesse nos pesquisadores ao redor do mundo, devido à sua possível utilização no processo de hidrólise enzimática de materiais lignocelulósicos visando à produção do etanol de segunda geração, tendo em vista a crescente busca por fontes de energia renováveis e novas tecnologias que possibilitem a autonomia energética dos países. Cabe lembrar que, para converter os carboidratos das cadeias de celulose ou hemicelulose da biomassa vegetal a açúcares fermentescíveis, é possível optar por rotas químicas ou enzimáticas. A via química é eficiente e rápida, mas gera resíduos tóxicos que devem ser tratados, aumentando o número de operações e consumindo mais energia, tornando o processo economicamente desfavorável. Já a via enzimática é mais lenta e tem como uma das principais barreiras processos eficientes e viáveis de produção de enzimas a custo que não inviabilize seu uso (Mishima et al., 2006).

A fermentação em estado sólido (FES), processo biotecnológico no qual uma matriz sólida porosa é fermentada geralmente por fungos que durante seu desenvolvimento no substrato secretam enzimas para o meio extracelular, é uma alternativa interessante para obtenção de enzimas celulolíticas a serem aplicadas na produção do bioetanol. A denominação de celulases designa uma classe de enzimas constituídas por endoglucanases (quebra a celulose na região amorfa e libera celo- 


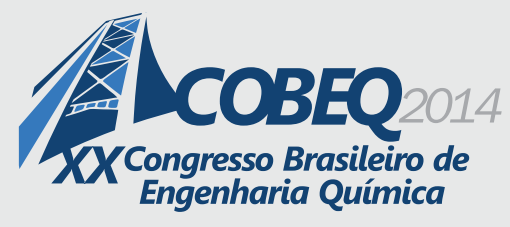

19 a 22 de outubro de 2014

Florianópolis/SC

oligossacarídeos), exoglucanases (quebra a celulose e celo-oligossacarídeos maiores e libera celobiose) e $\beta$-glicosidase (libera moléculas de glicose). No entanto, cada enzima produzida por microrganismos e processos diferentes poderá apresentar características particulares de atuação, o que pode determinar o controle de processo mais adequado para máximo aproveitamento da atividade catalítica da enzima de interesse. De um modo geral, a velocidade das reações enzimáticas, ou mais simplificadamente a atividade enzimática, varia com fatores diversos como concentração de enzima ou de substrato, temperatura, $\mathrm{pH}$ e efeito de inibidores ou ativadores enzimáticos (Nelson e Cox, 2003).

Ao comprovar experimentalmente a influência do $\mathrm{pH}$ na velocidade das reações enzimáticas, se obtém curvas que indicam que as enzimas apresentam um $\mathrm{pH}$ ótimo de atividade. $\mathrm{O} \mathrm{pH}$ pode afetar o desempenho da enzima de várias maneiras: a) o sítio ativo pode conter aminoácidos com grupos ionizados que podem variar com o $\mathrm{pH}$; b) a ionização de aminoácidos que não estão no sítio ativo pode provocar modificações na conformação da enzima; c) o substrato pode ver-se afetado pelas variações do $\mathrm{pH}$. As enzimas possuem grupos químicos ionizáveis nas cadeias laterais de seus aminoácidos. Dependendo do $\mathrm{pH}$ do meio, estes grupos podem ter carga elétrica positiva, negativa ou neutra. Como a conformação das proteínas depende em parte de suas cargas elétricas, haverá um $\mathrm{pH}$ no qual a conformação será a mais adequada para a atividade catalítica. Este é o chamado pH ótimo de atividade (Price et al., 1989).

A atividade catalítica das enzimas é altamente dependente da temperatura, como no caso dos catalisadores convencionais. No entanto, para enzimas, o aumento da temperatura provoca dois efeitos simultâneos: inicialmente, a taxa de reação aumenta, como se observa na maioria das reações químicas; com o aumento contínuo, a estabilidade da proteína decresce devido à desativação térmica, que consiste no rompimento das interações químicas responsáveis pela manutenção da estrutura terciária da proteína, responsável por sua atividade biológica (Furigo Jr. e Pereira, 2001). Desta forma, a temperatura influi na atividade enzimática e o ponto ótimo representa o máximo de atividade. Em temperaturas baixas, as enzimas encontram-se muito rígidas e, quando se supera um valor considerável (maior que $50^{\circ} \mathrm{C}$ ), a atividade cai bruscamente porque, como proteína, a enzima se desnatura. Em geral, os aumentos de temperatura aceleram as reações químicas: como regra geral, a cada $10^{\circ} \mathrm{C}$ de aumento, a velocidade de reação se duplica. As reações catalisadas por enzimas seguem esta lei geral. Entretanto, sendo proteínas, a partir de certa temperatura, as enzimas também começam a desnaturar-se pelo calor. A temperatura na qual a atividade catalítica é máxima chama-se temperatura ótima. Acima desta temperatura, o aumento de velocidade da reação devido ao aumento da temperatura é compensado pela perda de atividade catalítica devido à desnaturação térmica, e a atividade enzimática decresce rapidamente até anular-se (Madigan, 2004).

Neste contexto, o objetivo do presente trabalho foi avaliar, por meio da atividade de endoglucanase (CMCase), o $\mathrm{pH}$ e a temperatura ótimos dos extratos celulolíticos brutos produzidos pelos fungos termofílico Myceliophtora thermophila I-1D3b e mesofílico Trichoderma reesei por cultivo sólido em bagaço de cana e farelo de trigo $(7: 3$ e 9:1 m/m). O fungo Trichoderma reesei está entre os microrganismos com maiores potenciais para a produção de celulases e o fungo Myceliophtora thermophila I-1D3b é reportado na literatura como produtor de altos níveis de enzimas celulolíticas termoestáveis no substrato empregado (Zanelato et al., 2012), de modo que o extrato 


\section{9 a 22 de outubro de 2014 \\ Florianópolis/SC}

celulolítico cujo pH e temperatura ótimos são estudados apresentem alto potencial de aplicação para a hidrólise enzimática da biomassa vegetal.

\section{MATERIAIS E MÉTODOS}

Os ensaios foram realizados no Laboratório de Biorreatores do Departamento de Engenharia e Tecnologia de Alimentos do IBILCE/UNESP.

\subsection{Microrganismos e substratos}

Foram empregados os fungos Myceliophthora thermophila I-1D3b (termofílico) e Trichoderma reesei QM9414 (mesofílico). Para utilização, a cultura de Myceliophthora thermophila foi repicada em erlenmeyers contendo Agar Sabouraud Dextrose inclinados, mantidos em câmara de cultura BOD por 48 horas a $45{ }^{\circ} \mathrm{C}$; similarmente, a cultura de Trichoderma reesei foi repicada em Agar Batata Dextrose inclinados, mantidos a $28{ }^{\circ} \mathrm{C}$ por 168 horas. Os esporos foram suspensos com $100 \mathrm{~mL}$ de solução nutriente. A solução inoculante foi enriquecida com sais minerais (Zanelato et al., 2012). Uma alíquota foi retirada da suspensão para contagem de esporos em câmara de Neubauer. Um $\mathrm{mL}$ da suspensão ajustada para $10^{7}$ esporos/mL foi usada para inocular os sacos de fermentação.

Como substratos, foram empregados bagaço de cana e farelo de trigo. O bagaço foi doado pela Usina Vale, de Onda Verde-SP, e o farelo foi comprado no comércio local. O bagaço de cana foi lavado para remoção de açúcares. Ambos os materiais foram secos em estufa a $60{ }^{\circ} \mathrm{C}$ até peso constante, sendo posteriormente acondicionados em sacos de polietileno de parede espessa em câmara de refrigeração até utilização.

\subsection{Ensaios fermentativos em sacos de polipropileno}

Os ensaios fermentativos foram realizados em sacos de polipropileno de $12 \mathrm{~cm} \mathrm{x} 20 \mathrm{~cm}$ acoplados com bocal de PVC de $3,6 \mathrm{~cm}$ de diâmetro, os quais foram tampados com tampão de algodão envolto por gaze, a fim de garantir a troca de gases e assegurar que não houvesse contaminação por outros microrganismos. Os tubos de PVC foram fixados aos sacos com fita adesiva, para garantir que não ocorresse troca de gases por outro local que não os tampões. Dentro de cada saco fermentador, foi colocado um arame na forma de espiral para facilitar a aeração e evitar a aglomeração do substrato, auxiliando no processo fermentativo.

Em cada saco plástico, foram colocados $5 \mathrm{~g}$ de substrato composto por bagaço de cana e farelo de trigo na proporção de $7: 3 \mathrm{~m} / \mathrm{m}$ com $80 \%$ de umidade em base úmida para o fungo Myceliopthora thermophila I-1D3b (Zanelato et al., 2012) e $9: 1 \mathrm{~m} / \mathrm{m}$ com $70 \%$ de umidade para o fungo Trichoderma reesei QM9414 (Casciatori, 2012). O substrato foi inoculado com a suspensão fúngica e sua umidade foi acertada com solução nutriente composta por $0,35 \%$ de $\left(\mathrm{NH}_{4}\right)_{2} \mathrm{SO}_{4}, 0,3 \%$ de $\mathrm{KH}_{2} \mathrm{PO}_{4}, 0,05 \%$ de $\mathrm{MgSO}_{4}$ x 7H $\mathrm{H}_{2} \mathrm{O}, 0,05 \%$ de $\mathrm{CaCl}_{2}$ e $0,1 \%$ de Tween 20, pH 5,0.

Ao final do processo fermentativo, a extração das enzimas foi feita por água destilada (20 mL / $1 \mathrm{~g}$ de substrato seco, em câmara de agitação orbital, por $30 \mathrm{~min}$ a $100 \mathrm{rpm}$ ). O extrato foi filtrado e centrifugado $\left(10000 \mathrm{rpm}, 15 \mathrm{~min}, 5^{\circ} \mathrm{C}\right)$, após o que o sobrenadante foi empregado como solução 
enzimática bruta para os testes de atividade enzimática, sendo mantidos congelados até o momento das análises.

\subsection{Determinação da atividade enzimática}

A atividade celulolítica de endoglucanase (CMCase) foi tomada como base para caracterização físico-química do extrato celulolítico. A atividade sobre a CMC foi determinada a partir de reações contendo $0,1 \mathrm{~mL}$ da solução enzimática e $0,9 \mathrm{~mL}$ de solução de carboximetilcelulose (CMC - Sigma) a $1 \%$ em solução tamponante $\mathrm{NaOH} / a ́ c i d o$ acético a $0,1 \mathrm{~mol} / \mathrm{L}$.

A reação deu-se em banho termostático durante 10 minutos, sendo interrompida pela adição de $1,0 \mathrm{~mL}$ do reagente DNS (ácido1-3-dinitrosalicílico), como proposto por Miller (1959) para determinação do teor de açúcares redutores liberados na reação pelo ataque da enzima à estrutura da CMC. A solução foi mantida em água fervente por 10 minutos, após o que foi transferida para banho de gelo até o resfriamento da amostra.

Posteriormente, foram adicionados $8,0 \mathrm{~mL}$ de água destilada à solução e, em seguida, foi feita a leitura da absorbância em espectrofotômetro a $540 \mathrm{~nm}$, e a atividade foi calculada com base na curva padrão de glicose. Uma unidade de atividade endoglucanase foi definida como a quantidade de enzima necessária para liberar 1,0 $\mu$ mol de glicose por minuto de reação por $\mathrm{mL}$ de extrato enzimático. Em se tratando de fermentação em estado sólido, a atividade de endoglucanase foi obtida em unidade de atividade por grama de substrato sólido segundo a Equação 1.

$$
\frac{U}{g s s}=\frac{A b s+A}{B} \cdot \frac{1}{t} \cdot \frac{1}{V} \cdot D \cdot f
$$

onde Abs é a absorbância lida em 540 nm; A é o coeficiente linear da curva padrão de glicose; B é o coeficiente angular da curva padrão de glicose; $t$ é o tempo de reação em minutos; V é o volume de enzima em $\mathrm{mL}$; $\mathrm{D}$ é a diluição do extrato enzimático e f é a razão entre o volume de água utilizado na extração e a massa de substrato sólido.

\subsection{Caracterização físico-química da CMCase}

Para verificação do $\mathrm{pH}$ e da temperatura ótimos de atividade da enzima, foi utilizado um planejamento estatístico do tipo um fator de cada vez, sendo os fatores independentes o $\mathrm{pH}$ e a temperatura da reação. Assim, para cada extrato, o $\mathrm{pH}$ foi variado de $0,5 \mathrm{em} 0,5$ unidades desde $\mathrm{pH}$ 4,0 até $\mathrm{pH} 8,0$ a temperaturas fixas de $45^{\circ} \mathrm{C}$ para as enzimas dos fungos mesofílicos e $60{ }^{\circ} \mathrm{C}$ para as enzimas dos termofílicos. Os resultados das atividades CMCase foram submetidos a análise de variância (ANOVA), e o pH ótimo foi determinado através de comparação das médias das triplicatas por teste de Tukey, o que foi feito com auxílio do software Minitab ${ }^{\circledR} 15.1$. 


\section{9 a 22 de outubro de 2014 \\ Florianópolis/SC}

Uma vez determinado o pH ótimo, os ensaios para determinação da temperatura ótima foram realizados neste valor de $\mathrm{pH}$, variando-se a temperatura da reação de 5 em $5{ }^{\circ} \mathrm{C}$ desde $45{ }^{\circ} \mathrm{C}$ (mesofílicos) ou $55^{\circ} \mathrm{C}$ (termofílicos) até $85^{\circ} \mathrm{C}$. Os novos resultados das atividades CMCase também foram submetidos a análise de variância (ANOVA), e a temperatura ótima foi determinada através de comparação das médias por teste de Tukey. Sendo a análise do tipo um fator de cada vez, não foi avaliado efeito de interação entre as variáveis $\mathrm{pH}$ e temperatura.

\section{RESULTADOS E DISCUSSÕES}

\subsection{Temperatura e pH ótimos para atividade CMCase do extrato enzimático produzido pelo fungo Myceliophtora thermophila}

As condições de fermentação empregadas para obtenção do extrato enzimático do fungo Myceliophtora thermophila foram as condições ótimas já determinadas por Zanelato et al (2012), quais sejam: proporção de bagaço de cana e farelo de trigo $7: 3 \mathrm{~m} / \mathrm{m}$, umidade inicial do substrato 80 $\%$, temperatura de incubação $45^{\circ} \mathrm{C}$ e tempo de fermentação 144 horas.

Na Figura 1a, são apresentados os resultados de atividade CMCase em função do pH, que variou de 4,0 a 8,0. É possível observar que a curva de atividade CMCase foi ascendente até pH entre 5,0 e 5,5, quando atingiu o valor máximo e passou a declinar, inferindo-se que foi atingido o $\mathrm{pH}$ ótimo de atuação da enzima em um valor próximo a 5,5. Na Figura 1a, os pontos representam os resultados experimentais (média das triplicatas), enquanto a linha em vermelho representa o ajuste a uma curva de Gauss $\left(\mathrm{r}^{2}=0,83\right)$, que indica que o ponto de máxima atividade foi o valor de $\mathrm{pH}=5,3 \pm$ 0,1. De acordo com análise de variância dos dados (ANOVA one-way), constatou-se que de fato o $\mathrm{pH}$ tem efeito significativo sobre a atividade CMCase, com os maiores valores sendo obtidos em pH 5,0 e 5,5 .

$\mathrm{Na}$ Figura 1b, são apresentados os resultados de atividade CMCase em função da temperatura, no $\mathrm{pH}$ ótimo determinado anteriormente. Como o fungo é termofílico, a faixa de temperaturas analisadas variou de 55 a $85^{\circ} \mathrm{C}$. É possível observar que a atividade CMCase permaneceu praticamente constante de 55 até $75^{\circ} \mathrm{C}$, apresentando seu valor máximo a $65^{\circ} \mathrm{C}$. A partir de $80{ }^{\circ} \mathrm{C}$, a atividade enzimática do extrato passou a declinar, inferindo-se que foi atingida a temperatura de desnaturação da enzima. De acordo com análise de variância dos dados, constatou-se que a temperatura de fato exerce efeito significativo sobre a atividade CMCase, com os maiores valores sendo obtidos na faixa de 55 a $75^{\circ} \mathrm{C}$, mas notando-se queda significativa da atividade a partir de 80 ${ }^{\circ} \mathrm{C}$.

Considerando-se que a atividade celulolítica do extrato produzido pelo fungo termofílico Myceliophtora sp. começou a decair significativamente somente a partir de $75^{\circ} \mathrm{C}$, o extrato pode ser considerado termofílico, uma vez que apresenta atividade a temperaturas maiores que $60{ }^{\circ} \mathrm{C}$. Da Figura 1b, é possível inferir ainda que a atividade CMCase manteve-se elevada em ampla faixa de temperaturas, o que é interessante do ponto de vista de um processo industrial, pois permite uma flexibilização das condições térmicas da operação. 

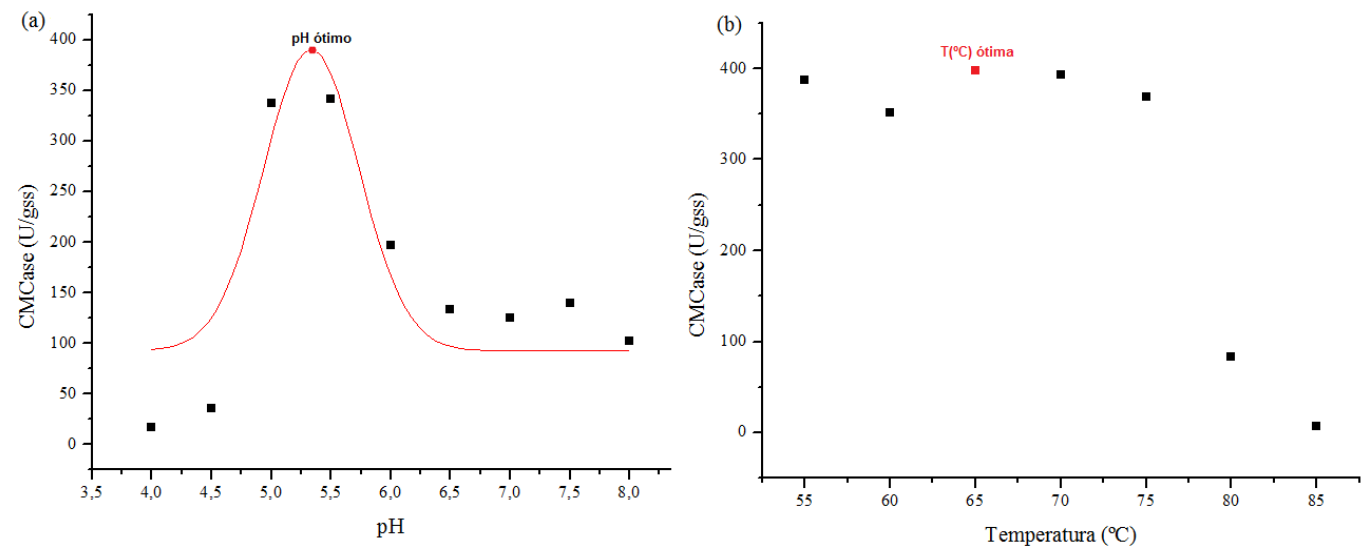

Figura 1 - Atividade de CMCase do extrato produzido pelo fungo Myceliophtora thermophila em função (a) do pH; (b) da temperatura.

\subsection{Temperatura e pH ótimos para atividade CMCase do extrato enzimático produzido pelo fungo Trichoderma reesei}

As condições de fermentação empregadas para obtenção do extrato enzimático do fungo Trichoderma reesei foi proporção de bagaço de cana e farelo de trigo 9:1 m/m, umidade inicial do substrato $70 \%$, temperatura de incubação $28^{\circ} \mathrm{C}$ e tempo de fermentação 168 horas ou sete dias, que foram a temperatura e o tempo de cultivo empregados por Pinto (2010).

Na Figura 2a, são apresentados os resultados de atividade CMCase em função do pH, que variou de 4,0 a 8,0. É possível observar que a curva de atividade $\mathrm{CMCase}$ foi ascendente até $\mathrm{pH}=4,5$, quando atingiu o valor máximo e passou a declinar, inferindo-se que foi atingido o $\mathrm{pH}$ ótimo de atuação da enzima. Na Figura 2a, os pontos representam os resultados experimentais (média das triplicatas), enquanto a linha em vermelho representa o ajuste a uma curva de Gauss $\left(\mathrm{r}^{2}=0,90\right)$, que indica que o ponto de máxima atividade foi o valor de $\mathrm{pH}=4,5 \pm 0,1$.
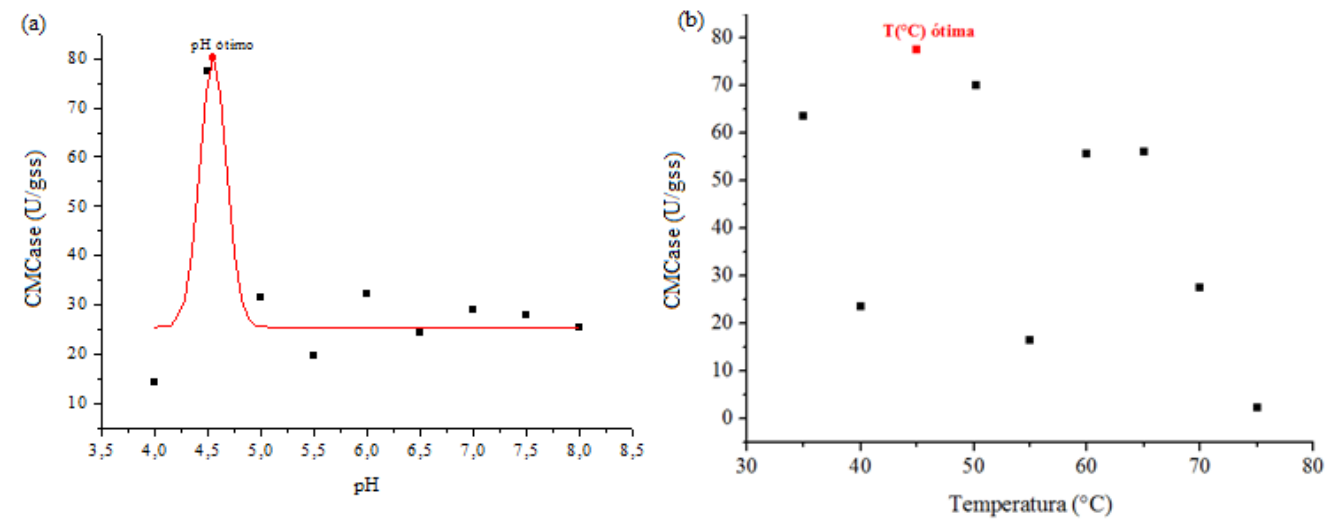

Figura 2 - Atividade de CMCase do extrato produzido pelo fungo Trichoderma reesei em função em função (a) do pH; (b) da temperatura. 


\section{9 a 22 de outubro de 2014 \\ Florianópolis/SC}

De acordo com análise de variância dos dados, constatou-se que de fato o $\mathrm{pH}$ tem efeito significativo sobre a atividade CMCase do extrato produzido pelo $T$. reesei, com o valor significativamente maior sendo obtido em $\mathrm{pH}$ 4,5. Em $\mathrm{pHs}$ mais básicos, as atividades não apresentaram diferença significativa, mas diferiram significativamente da atividade a $\mathrm{pH} 4,0$. Ressalte-se que, dentre os extratos avaliados neste trabalho, o extrato celulolítico produzido pelo fungo $T$. reesei é o que apresenta atividade CMCase ótima em $\mathrm{pH}$ mais ácido, inferior a 5,0, concordando com as recomendações da literatura para análise da atividade celulolítica do extrato enzimático produzido por este fungo (GHOSE, 1987).

Ainda com relação ao formato da curva de atividade CMCase em função do pH, observa-se que o pico de máxima atividade do extrato produzido pelo $T$. reesei foi mais estreito que o pico observado para o Myceliophtora thermophila, indicando que o extrato produzido pelo T. reesei é mais sensível ao $\mathrm{pH}$, uma vez que a variação, para mais ou para menos, de apenas 0,5 unidade de $\mathrm{pH}$ pode alterar significativamente a atividade CMCase do extrato.

Além disso, observa-se que este fungo apresenta menores atividades CMCase que o termofílico Myceliophtora thermophila, indicando que a produção de endoglucanases é baixa, apesar de este ser um fungo tradicionalmente empregado para produção de celulases. De acordo com Lynd et al. (2002), as endoglucanases, quantificadas pela atividade CMCase, representam apenas $20 \%$ do total de celulases produzidas pelo fungo $T$. reesei. Tais observações reiteram que os estudos não devem se limitar aos ensaios de atividades enzimáticas, sendo conveniente, em algum momento, empregar as enzimas na sacarificação do material celulósico que realmente deverá ser utilizado para obtenção do bioetanol, com o intuito de avaliar a real capacidade de hidrólise da celulose pela enzima.

Na Figura 2b, são apresentados os resultados de atividade CMCase em função da temperatura. Como o fungo é mesofílico, a faixa de temperaturas analisadas foi de 35 a $75{ }^{\circ} \mathrm{C}$. Não foi possível observar uma tendência muito definida da atividade CMCase em função da temperatura do banho termostático, sendo possível notar que a máxima atividade foi atingida nas temperaturas de 45 e 50 ${ }^{\circ} \mathrm{C}$. Observa-se ainda que a atividade CMCase foi praticamente nula para análise do extrato acima de $70{ }^{\circ} \mathrm{C}$, indicando que a enzima provavelmente já estaria desnaturada.

De acordo com análise de variância dos dados, constatou-se que a temperatura de fato exerce efeito significativo sobre a atividade CMCase do extrato produzido pelo $T$. reesei, com os maiores valores sendo obtidos na faixa de 45 a $60{ }^{\circ} \mathrm{C}$, notando-se queda significativa da atividade a partir de $75^{\circ} \mathrm{C}$, onde o extrato apresentou atividade praticamente nula. Assim, pode-se dizer que a atividade CMCase deste extrato se manteve pouco afetada pela temperatura na faixa de 35 a $70^{\circ} \mathrm{C}$, após o que houve desnaturação da enzima.

\section{CONCLUSÃO}

A ótima atividade endoglucanase do extrato enzimático produzido pelo fungo mesofílico Trichoderma reesei foi obtida em $\mathrm{pH} 4,5$ e temperatura igual a $45{ }^{\circ} \mathrm{C}(80 \mathrm{U} / \mathrm{g})$, sendo a enzima inativada a partir de $70{ }^{\circ} \mathrm{C}$. Para o extrato produzido pelo fungo termofílico Myceliophthora thermophila, a ótima atividade endoglucanase foi obtida em $\mathrm{pH} 5,5$ e temperatura igual a $65^{\circ} \mathrm{C}(350$ 
$\mathrm{U} / \mathrm{g}$ ), e somente a partir de $80^{\circ} \mathrm{C}$ a atividade endoglucanase declinou, sugerindo que as celulases de fungos termofílicos são promissoras para aplicação na cadeia de produção do etanol de segunda geração, pois manteve-se elevada em uma ampla faixa de temperaturas, o que é interessante do ponto de vista industrial, já que permite uma flexibilização nas condições térmicas do processo.

\section{REFERÊNCIAS}

CASCIATORI, P.A. Produção de Enzimas Celulolíticas Associada à Cinética de Crescimento dos Fungos Thermoascus aurantiacus e Trichoderma reesei em Fermentação no Estado Sólido. Trabalho de Conclusão de Curso - Bacharelado em Química Ambiental, Universidade Estadual Paulista, São José do Rio Preto, 2012.

FURIGO JR, A.; PEREIRA, E. B. Enzimas e suas aplicações - Cinética enzimática. 2001. 39f. (Apostila) Universidade Federal de Santa Catarina, Florianópolis, 2001.

GHOSE, T. K. Measurement of Cellulase Activities. Pure Appl. Chem., v. 59, p. 257-268, 1987.

LYND, L. R.; WEIMER, P. J.; ZYL, W. H.; PRETORIUS, I. S. Microbial cellulose utilization: Fundamentals and Biotechnology. Microbiol Mol Biol Rev., v. 66, p. 506-577, 2002.

MADIGAN, M. T.; MARTINKO, J. M.; PARKER, J. Brock Biology of Microorganisms. New York: Editora Prentice Hall International Inc. 10ed. 2004.

MILLER, G. L. Use of Dinitrosalicylic Acid Reagent for Determination of Reducing Sugar. Anal. Chem., v. 31, p. 426-428, 1959.

MISHIMA, D.; TATEDA, M.; IKE, M.; FUJITA, M. Comparative study on chemical pretreatments to accelerate enzymatic hydrolysis of aquatic macrophyte biomass used in water purification processes. Bioresour. Technol., v. 97, p. 2166-2172, 2006.

NELSON, D. L.; COX, M. Carbohydrates and Glycobiology. In: Lehninger Principles of Biochemistry, 4ed., Palgrave Macmillan, cap. 7, p. 249, 2003.

PINTO, T. O. P. Produção de Enzimas Celulolíticas pelos fungos Thermoascus aurantiacus CBMAI 756, Thermomyces lanuginosus, Trichoderma reesei QM9414 e Penicillium viridicatum RFC3 e Aplicação na sacarificação do bagaço de cana de açúcar com diferentes pré-tratamentos. 2010. 91f. Dissertação (Mestrado). Universidade Estadual Paulista, São José do Rio Preto - SP, 2010.

PRICE, N. C.; STEVENS, L. Fundamentals of Enzymology. 2ed. Oxford Science Publications, 1989.

ZANELATO, A.I.; SHIOTA, V.M.; GOMES, E.; THOMÉO, J.C. Endoglucanase production with the newly isolated Myceliophtora sp. $\mathrm{i}-1 \mathrm{~d} 3 \mathrm{~b}$ in a packed bed solid state fermentor. Braz. J. Microbiol., v. 43, n. 4, p. 1536 - 1544, 2012. 